\title{
Nuclear structure effects in quasifission - understanding the formation of the heaviest elements
}

\author{
D. J. Hinde ${ }^{1, a}$, E. Williams ${ }^{1}$, G. Mohanto ${ }^{1, b}$, C. Simenel ${ }^{1}$, D. Y. Jeung ${ }^{1}$, M. Dasgupta ${ }^{1}$, E. Prasad ${ }^{1}$, A. Wakhle $^{1, c}$, K. $^{2}$ \\ Vo-Phuoc ${ }^{1}$, I. P. Carter ${ }^{1}$, K. J. Cook ${ }^{1}$, D. H. Luong ${ }^{1}$, C. S. Palshetkar ${ }^{1, d}$, D. C. Rafferty ${ }^{1}$, and E. C. Simpson ${ }^{1}$ \\ ${ }^{1}$ Department of Nuclear Physics, Research School of Physics and Engineering, The Australian National University, Canberra, \\ ACT 2601, Australia
}

\begin{abstract}
Quasifission is an important process suppressing the fusion of two heavy nuclei in reactions used to create superheavy elements. Quasifission results in rapid separation of the dinuclear system initially formed at contact. Achieving reliable a priori prediction of quasifission probabilities is a very difficult problem. Through measurements with projectiles from $\mathrm{C}$ to Ni, the Australian National University's Heavy Ion Accelerator Facility and CUBE spectrometer have been used to map out mass-angle distributions (MAD) - the fission mass-ratio as a function of centre-of-mass angle. These provide information on quasifission dynamics in the least modeldependent way. Average quasifission time-scales have been extracted, and compared with TDHF calculations of the collisions, with good agreement being found. With the baseline information from the survey of experimental MAD, strong influences of the nuclear structure of the projectile and target nuclei can be clearly determined.
\end{abstract}

\section{Introduction}

Fusion of two heavy nuclei has been a successful pathway to produce new very heavy and superheavy elements (SHE). However, the production yield of SHE is very significantly suppressed [1] by quasifission [2]. This dynamical non-equilibrium process results when the combined system formed after capture separates before a compact compound nucleus is formed, resulting in two (fissionlike) fragments. The probability of quasifission $\left(P_{Q F}\right)$ can be very large, with the complementary probability of compound nucleus formation $\left(P_{C N}=1-P_{Q F}\right)$ being possibly lower than $10^{-6}$ in unfavourable reactions. Understanding the competition between quasifission and fusion is thus crucial to predict the best fusion reactions to use to form new isotopes of heavy and super-heavy elements. The months of beamtime needed to determine if a given reaction is likely to be successful or not gives additional motivation to obtain a reliable predictive model based on an full understanding of this competition.

\section{Mass-Angle Distributions}

The measurement of all binary mass-splits (characterised by the mass ratio $M_{R}=M_{1} /\left(M_{1}+M_{2}\right)$ between the initial projectile mass $M_{1}$ and the target mass $M_{2}$, when determined over a wide range of scattering angles, results

\footnotetext{
ae-mail: david.hinde@anu.edu.au

${ }^{\mathrm{b}}$ Current address: BARC, Mumbai, India

${ }^{\mathrm{c}}$ Current address: National Superconducting Cyclotron Laboratory,

MSU, East Lansing, Michigan 48824, USA

${ }^{\mathrm{d}}$ Current address: TIFR, Mumbai, India
}
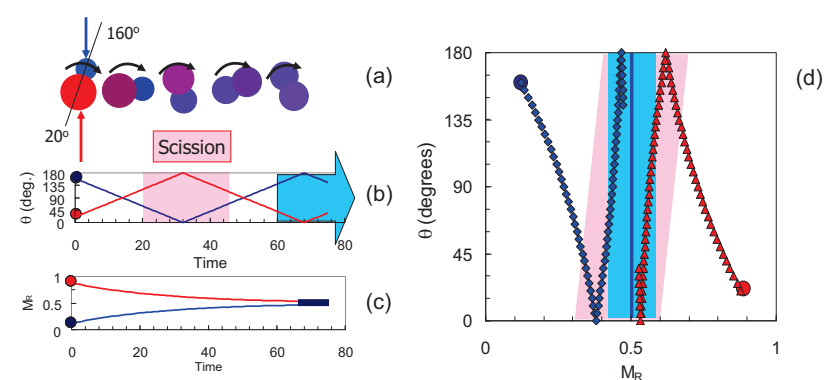

Figure 1. The relationship between sticking time and the massangle distribution. For the reaction sequence sketched in (a), the time dependence (arbitrary units) of angle (b) and mass-ratio (c) are shown. Such short times cannot be measured directly, but angle and mass-ratio can. The individual mass and angle dependencies combine to give a trajectory (d) on the MAD for a single impact parameter. Including a range of impact parameters, scission after half a turn (pink) or a full turn (blue) will give strong or weak mass-angle correlations respectively.

in a two-dimensional matrix, referred to as a mass-angle distribution (MAD). The relationship of the MAD to the "sticking time" between capture and scission is illustrated schematically in Fig. 1. The projectile nucleus (blue) is incident from the top of the page, and sticks to the larger target nucleus (red). The system then rotates, Fig. 1(b) illustrating schematically angle against time (in arbitrary units) for a single angular momentum value; in reality a distribution will be present. For a parabolic potential, masssymmetry is approached with an expected time dependence $1-\exp \left(\frac{t}{\tau_{e q}}\right)$, where $\tau_{e q}$ is the mass-equilibration time 


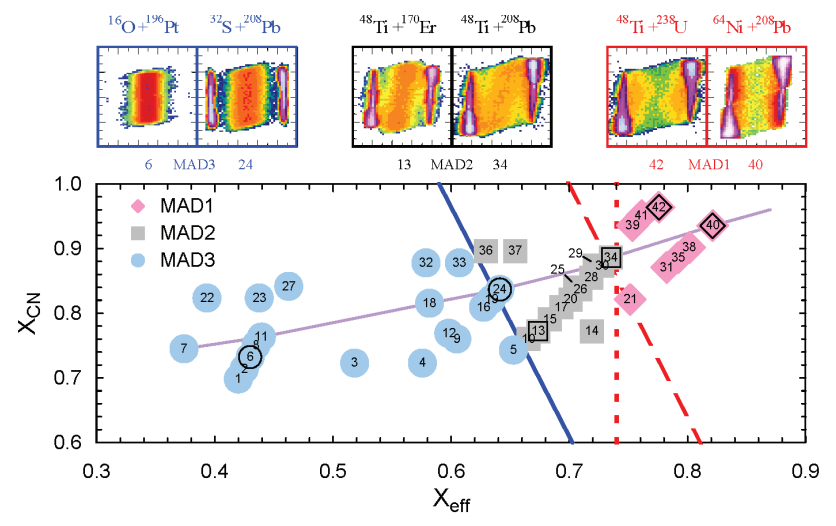

Figure 2. The numbers surrounded by a symbol indicating the classification of MAD observed refer to the reaction number in Ref. [3], and are plotted as a function of the effective fissility parameter in the entrance channel $x_{\text {eff }}$ and the compound nucleus fissility parameter $x_{C N}$ (see text). The blue diagonal full line represents the empirical boundary between reactions with no massangle correlation (to the left) and those that have (right). The thin line represents the locus of reactions with ${ }^{208} \mathrm{~Pb}$ (see text). Examples of MAD are shown in the panels above.

constant [2]. This dependence is sketched in Fig. 1(c). As a dynamical out-of-equilibrium process, the time scale information carried by the MAD gives key information on the dynamics.

It has been found that the quasifission probability and MAD characteristics can depend strongly on the nuclear structure of the two colliding nuclei. This may not be surprising, since nuclear fusion requires the merger of two individual quantum systems, each with their own individual shell structure. In particular, heavy statically deformed nuclei show [4-7] suppression of fusion when the long axis is aligned with the contact angle of the lighter nucleus (called deformation alignment). This condition applies in collisions at sub-barrier energies. In contrast, collisions of spherical magic nuclei at sub-barrier energies can result in enhancement of heavy element yields $[8,9]$ and fission distributions characteristic of fusion-fission [10, 11].

To improve our quantitative understanding of the role of shell structure in the dynamics of quasifission, we make an analogy with the liquid drop model approach to nuclear masses, in which localized shell effects can be quantified when the underlying smooth (liquid drop) trends are well defined. To define the smooth trends in quasifission, a large number of MAD measurements have been selected from measurements recently made at the ANU [3-5, 1117]. By investigating empirically those nuclear stucture variables that affect quasifission, the ultimate goal to have a reliable predictive model of quasifission, including all relevant physics, will be a step closer.

During their development, such models must be tested. To test by comparing with measured superheavy element cross sections is appealing. However, comparison with measured characteristics of the competing quasifission process seems more reliable: if a model can't reproduce the quasifission characteristics, which may comprise $99 \%$ or more of the cross section, it may throw doubt on the reliability of predictions of the fusion probability, which makes up the remaining $1 \%$, or even less.

To determine the smooth trends in quasifission dynamics, we map the reaction outcomes (defined in terms of the MAD classes discussed below) against variables that reflect the balance between nuclear and Coulomb forces during the collision. These are expected to determine the dynamics in reactions forming very heavy elements. According to the characteristics of the MAD (minimum mass yield at symmetry with mass-angle correlation, mass-angle correlation with peak yield at symmetry, and no significant mass-angle correlation), they are assigned as type MAD1, MAD2 and MAD3 respectively [3]. Note that it has recently been shown [18] that heavy-ion fusion-fission unexpectedly can show a mass-asymmetric (double-peaked) fission mass distribution, so it is the lack of correlation of mass with angle that characterises slow fission, rather than a peak at mass-symmetry. These MAD categories can be associated with characteristic sticking times $[3,16]$ of $\tau_{\mathrm{MAD} 1}<5 \cdot 10^{-21} \mathrm{~s}, \tau_{\mathrm{MAD} 2} \sim 5 \cdot 10^{-21} \mathrm{~s}$ and $\tau_{\mathrm{MAD} 3}>10 \cdot 10^{-21} \mathrm{~s}$ respectively.

It was proposed in Ref. [19] that there should be scaling behavior for reactions, based on the schematic "chaotic regime dynamics" model of fusion of heavy nuclei. This model also predicted the "extra-extra-push" kinetic energy [20] needed to overcome the unconditional saddlepoint energy for a given reaction. The two scaling parameters expected to strongly define the reaction outcome are (i) the effective fissility parameter of the entrance channel, $x_{\text {eff }}$ (relevant to necked shapes close to the generally mass-asymmetric contact configuration in the entrance channel), and (ii) the fissility parameter of the compound nucleus, $x_{C N}$ (applicable to shapes without a constricted neck [20] where the mass-asymmetry degree of freedom is not constrained). Experimental MAD outcomes are shown in Fig. 2. Importantly these are chosen for beam energies somewhat above the capture barrier (typically by $\sim 6 \%$ ). Here the experimentally observed effects of deformation alignment [4-7] and closed shells, seen in measurements at below-barrier energies [10, 11], are much reduced [5, 21, 22].

The MAD classes are clustered into groups, indicating the appropriateness of the scaling parameters. The boundary across which a mass-angle correlation becomes significant (between MAD classes 3 and 2) shows a dependence on both variables, with a stronger dependence on the entrance-channel than on the compound nucleus fissility. The full blue line is an estimate of this boundary based on current data. Mass-angle distributions for reactions on this line should show similar mass-angle correlations, associated with similar reaction trajectories and timescales. The same should be true for reactions on nearby parallel lines. The dashed and dotted red lines indicate the uncertainty in the boundary of reactions (MAD1) which do not show a peak at mass-symmetry in the angle-integrated distribution.

Such similarities in behaviour between reactions with similar fissilities, but different nuclear structure, is not 


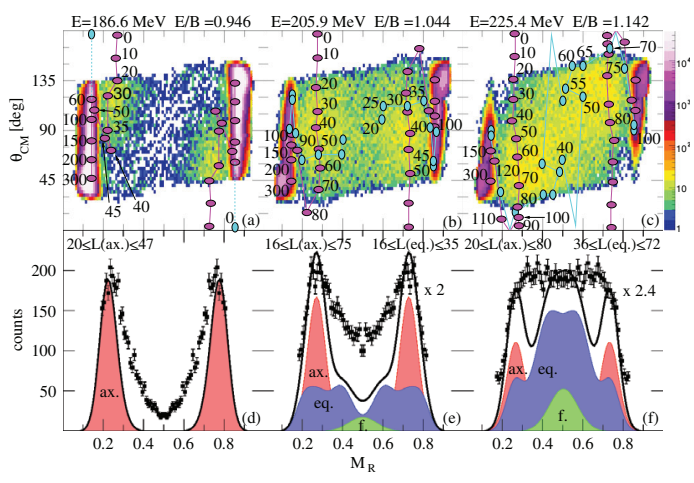

Figure 3. (a)-(c) Measured mass-angle distributions at centerof-mass energies $\mathrm{E}$ near the capture barrier for ${ }^{40} \mathrm{Ca}+{ }^{238} \mathrm{U}$. The horizontal and vertical ellipsoids show the final outcome of TDHF calculations for axial and equatorial orientations, respectively. The values of $\mathrm{L}$ are indicated near the points associated to the light (heavy) fragments for the axial (equatorial) orientation. (d)-(f) Projected mass ratio $M_{R}$ in the range $0.2<M_{R}<0.8$. The mass ratio distributions estimated from the TDHF results are shown by shaded areas for quasifission in the axial (ax.) and equatorial (eq.) configurations, and for fusion-fission (f.) events. The sum of these distributions is shown by the solid line for comparison with experiment.

found at sub-barrier energies. Here it is well-known that only collisions with the tips of deformed nuclei lead to capture [23], and that fission angular distributions [6], mass distributions $[4,15,24]$ and $\operatorname{MAD}[5,17]$ point to the changing nature (shorter sticking time) of quasifission under these circumstances. Extensive microscopic TDHF calculations of the outgoing masses and angles of binary events have shown a good match [17] to the experimental MAD, and thus to sticking times and mass evolution in the reaction ${ }^{40} \mathrm{Ca}+{ }^{238} \mathrm{U}$. This is illustrated in Fig. 3. This agreement extends throughout the transition from subbarrier (where contact occurs in the deformation-aligned orientation) to above-barrier energies (at which all collision orientations contribute), as seen in Fig. 3.

In sub-barrier reactions with closed-shell nuclei, it has been shown [11] that increasing the "magicity" in the entrance channel results in narrower mass distributions, correlated with a reduced mass-angle correlation, indicative of longer sticking times. For the ${ }^{48} \mathrm{Ca}+{ }^{208} \mathrm{~Pb}$ reaction, with four magic numbers, measured mass distributions [10] seem consistent with fusion-fission. These results led to the conclusion [11] that "magicity" plays the strongest role when the $N / Z$ values of the projectile and target nuclei are matched. When this is the case, transfer reactions that destroy entrance-channel magicity (as in the ${ }^{40} \mathrm{Ca}+{ }^{208} \mathrm{~Pb}$ reaction [11]) are minimized, preserving the closed shell nature of the collision partners as long as possible during the merging of the two nuclei.

Very recent ANU MAD measurements, for the reaction of isotopes of $\mathrm{Cr}$ with $\mathrm{Pb}$, are shown in Fig. 4. These measurements were at sub-barrier energies, resulting in the low excitation energies above the ground-state as indicated. They support the picture from the $\mathrm{Ca}+\mathrm{Pb}$ data.

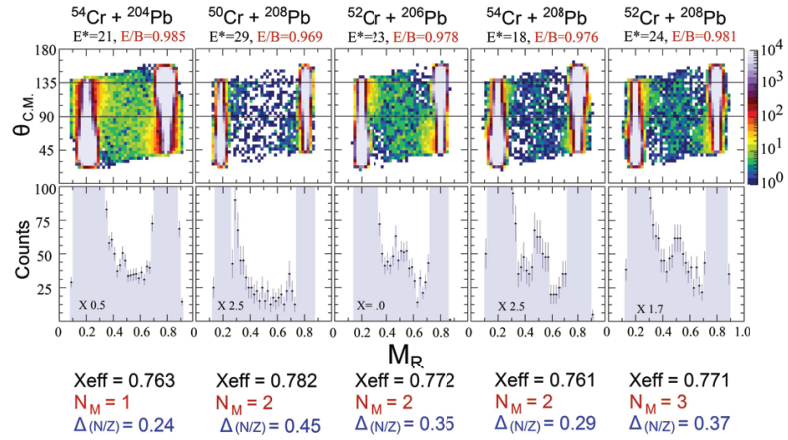

Figure 4. MAD and projected mass-ratio distributions for backward angles from $90^{\circ}$ to $135^{\circ}$ (as indicated in the MAD plots), for reactions of ${ }^{50,52,54} \mathrm{Cr}$ isotopes with ${ }^{204,206,208} \mathrm{~Pb}$. Sub-barrier beam energies (denoted by $E / B$ ), resulted in the low excitation energies $E^{*}$. The entrance channel fissility $x_{e f f}$, the number of entrance-channel magic numbers, and the mismatch in $N / Z$ values are indicated for each reaction (see text). The reaction outcome changes from a minimum in yield at mass-symmetry (left) to a narrow peak at symmetry, having no evidence of a massangle correlation.

The left three reactions all form the compound nucleus ${ }^{258} \mathrm{Sg}$. The difference between the $N / Z$ values of the target and projectile nuclei is denoted by $\Delta_{(N / Z)}$ in the figure. The reactions are ordered from less favourable to more favourable for fusion, first by the number of magic numbers in the entrance channel $\left(N_{M}\right)$, and then by $\Delta_{(N / Z)}$. The left-most reaction has only a single magic number in the entrance channel, and shows a U-shaped mass distribution, consistent with MAD class 1 . With two magic numbers, the reactions with $\Delta_{N / Z}$ closer to zero show a peak at masssymmetry, associated with an angle-independent ridge in the MAD. With three magic numbers, but less favourable

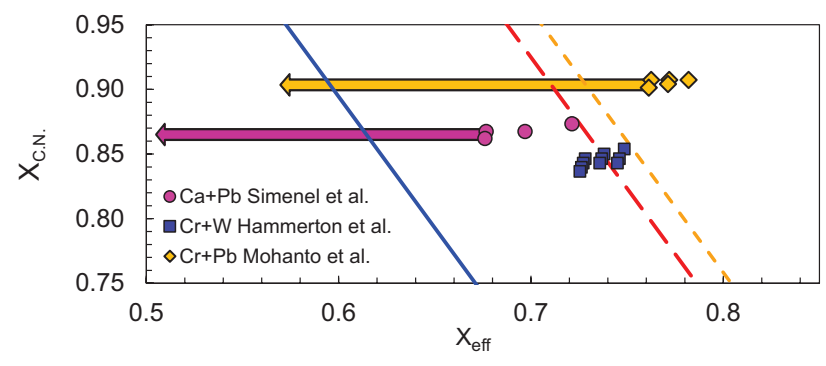

Figure 5. Location of recent measurements of MAD in reactions of isotopes of $\mathrm{Ca}$ with $\mathrm{Pb}, \mathrm{Cr}$ with $\mathrm{W}$ and $\mathrm{Cr}$ with $\mathrm{Pb}$, plotted as a function of $x_{e f f}$ and $x_{C N}$, as in Fig.2. In the absence of nuclear structure effects, the outcomes should be MAD2 and MAD1. The $\mathrm{Cr}+\mathrm{W}$ data [25], measured at above-barrier energies, fit well with expectations (but suggest that the MAD1-MAD2 boundary should be shifted slightly to the yellow dashed line). The ${ }^{48} \mathrm{Ca}+{ }^{208} \mathrm{~Pb}$ reactions show MAD consistent with fusion-fission, as indicated by the arrow. The most surprising outcome is a fraction of $\mathrm{Cr}+\mathrm{Pb}$ events (where MAD1 is expected) being consistent with MAD3. This suggests that there may be a significant bifurcation of trajectories between fast quasifission, and slow fission which may be consistent in its properties with fusion-fission. 
$\Delta_{N / Z}$, a similar result is seen. This mass-symmetric peak becomes a smaller and smaller fraction of the total fission yield (integrated between $0.3<M_{R}<0.7$ ), as the beam energy is increased. These data show a very similar behaviour to the ${ }^{40,44,48} \mathrm{Ca}+{ }^{204,208} \mathrm{~Pb}$ reactions [11]. However, the transition from a fully U-shaped mass distribution to a U-shaped distribution and a narrow mass-symmetric mass distribution is in some ways an even more drastic change in reaction outcome. It implies a bifurcation of trajectories not seen so clearly in any other measurement of this type. The very sudden change in outcome, and the bifurcation, which depends on magicity and $N / Z$ matching, will be a severe challenge for models of quasifission to reproduce. And yet it is this level of sensitivity that models must strive to reproduce, to optimise experimental opportunities to create new isotopes of superheavy elements.

\section{Acknowledgments}

We acknowledge NCRIS operations support for the ANU Heavy Ion Accelerator Facility, and ARC grants DP130101569, DP140101337, FL110100098, FT120100760 and DE140100784. Thanks to N. Lobanov, T. Kibedi and the accelerator staff for supporting use of the superconducting Linac accelerator.

\section{References}

[1] D. J. Hinde, M. Dasgupta and A. Mukherjee, Phys. Rev. Lett. 89, 282701 (2002).

[2] J. Tōke, B. Bock, G. X. Dai et al., Nucl. Phys. A 440, 327 (1985).

[3] R. du Rietz, E. Williams, D. J. Hinde et al., Phys. Rev. C 88, 054618 (2013).

[4] D. J. Hinde, R. du Rietz, M. Dasgupta et al., Phys. Rev. Lett. 101, 092701 (2008).

[5] D. J. Hinde, R. G. Thomas, R. du Rietz et al., Phys. Rev. Lett. 100, 202701 (2008).

[6] D. J. Hinde, M. Dasgupta, J. R. Leigh et al., Phys. Rev. C 53, 1290 (1996).
[7] D. J. Hinde, M. Dasgupta, J. R. Leigh et al., Phys. Rev. Lett. 74, 1295 (1995).

[8] D. J. Hinde and M. Dasgupta, Phys. Lett. B 622, 23 (2005).

[9] J. Khuyagbaatar, D. J. Hinde, I. P. Carter et al., Phys. Rev. C 91, 054608 (2015).

[10] E. V. Prokhorova, A. A. Bogachev, M. G. Itkis et al., Nucl. Phys. A 802, 45 (2008).

[11] C. Simenel, D. J. Hinde, R. du Rietz et al., Phys. Lett. B 710, 607 (2012).

[12] R. G. Thomas, D. J. Hinde, D. Duniec et al., Phys. Rev. C 77, 034610 (2008).

[13] R. Rafiei, R. G. Thomas, D. J. Hinde et al., Phys. Rev. C 77, 024606 (2008).

[14] R. du Rietz, D. J. Hinde, M. Dasgupta et al., Phys. Rev. Lett. 106, 052701 (2011).

[15] C. J. Lin, R. du Rietz, D. J. Hinde et al., Phys. Rev. C 85, 014611 (2012).

[16] E. Williams, D. J. Hinde, M. Dasgupta et al., Phys. Rev. C 88, 034611 (2013).

[17] A. Wakhle, C. Simenel, D. J. Hinde et al., Phys. Rev. Lett. 113, 182502 (2014).

[18] E. Prasad, D. J. Hinde, K. Ramachandran et al., Phys. Rev. C 91, 064605 (2015).

[19] J. Blocki, H. Feldmeier and W. Swiatecki, Nucl. Phys. A 459, 145 (1996).

[20] S. Bjornholm and W. Swiatecki, Nucl. Phys. A 391, 471 (1982).

[21] K. Nishio, H. Ikezoe, S. Mitsuoka et al., Phys. Rev. C 77, 064607 (2008).

[22] I. M. Itkis, E. M. Kozulin, M. G. Itkis et al., Phys. Rev. C 83, 064613 (2011).

[23] M. Dasgupta, D. J. Hinde, N. Rowley et al., Annu. Rev. Nucl. Part. Sci. 48, 401 (1998).

[24] K. Nishio, H. Ikezoe, I. Nishinaka et al., Phys. Rev. C 82, 044604 (2010).

[25] K. Hammerton, Z. Kohley, D. J. Hinde et al., Phys. Rev. C 91, 041602 (2015). 